\title{
ALGEBRAIC SURFACES AND 4-MANIFOLDS: SOME CONJECTURES AND SPECULATIONS
}

\author{
ROBERT FRIEDMAN AND JOHN W. MORGAN
}

Introduction. Since the time of Riemann, there has been a close interplay between the study of the geometry of complex algebraic curves (or, equivalently, compact Riemann surfaces) and the topology of 2-manifolds. These connections arise from the uniformization theorem, which asserts that every simply connected Riemann surface is conformally equivalent to either the Riemann sphere, the plane, or the interior of the unit disk. From this it follows that every compact Riemann surface has a conformally equivalent metric of constant curvature. A key idea in the proof of this result is the Dirichlet problem: Find a harmonic function on a Jordan region $R$ in the plane with given boundary values. Any such harmonic function minimizes the functional

$$
f \mapsto \iint_{R}|\nabla f|^{2} d x d y
$$

among all functions on $R$ with the given boundary values. The existence of such functions has a physical interpretation. If we view the boundary values as a charge density on $\partial R$ then the harmonic function corresponds to the resulting electrostatic potential in $R$. This physical interpretation suggests that such a harmonic function should exist and should be unique, at least for reasonable regions and boundary conditions. In fact one can solve this problem by integrating over the boundary a family of Green's functions, each of which is the electrostatic potential of a point charge, against the charge density.

The connection between the analysis and differential geometry of 2-dimensional metrics of constant curvature on the one hand and the topology and algebraic geometry of compact Riemann surfaces on the other has been a fruitful one. Major ideas have evolved from the work of Teichmüller [39], and Ahlfors and Bers $[\mathbf{1}, 6]$ and more recently through the work of Thurston [40]. Some of the nicest recent examples of this interplay can be found in [20], with applications to the algebraic geometry in [21].

Of course a smooth complex algebraic variety of dimension $n$ is naturally a $C^{\infty}$-manifold of dimension $2 n$. But for $n \geq 3$ the algebraic geometry of these varieties diverges quite markedly from the topology for many reasons, some of which will be made more precise below. Quite surprisingly, very deep connections have emerged recently between the complex geometry of a complex

Received by the editors April 22, 1987 and, in revised form, June 1, 1987.

1980 Mathematics Subject Classification (1985 Revision). Primary 57R55, 14J15.

First author supported by NSF grant DMS 85-03743 and Alfred E. Sloan Foundation; second author supported by NSF grant DMS 85-03758. 
surface and the $C^{\infty}$-topology of the underlying 4-manifold. These connections follow the paradigm briefly indicated above in the case of Riemann surfaces. By minimizing a certain functional analogous to the Dirichlet integral one constructs geometric objects (Yang-Mills connections) associated to Riemannian 4-manifolds. These should be thought of as "harmonic" connections. They have (at least sometimes) a physical interpretation in terms of three of the basic forces of nature (the electromagnetic, the weak, and the strong force). The original emphasis for the analysis of these objects came from the physics. Yang and Mills introduced nonabelian gauge theory in 1954 [46]. There was an upsurge of interest in solving the Yang-Mills equations in the 1970s, when, after initial work of Penrose, Atiyah became interested in the problem. It was the insight of Penrose and Atiyah that techniques of algebraic geometry might produce physically interesting solutions to these equations (cf. [2, 34]).

While the original applications of this confluence of physics and mathematics were from topology and algebraic geometry to the physical problems, there was eventually a reversal of direction, as often happens in mathematics. It was Donaldson [11] who realized that the spaces of Yang-Mills connections could be used to define invariants to study 4 -manifolds and complex surfaces. These recent advances and the subsequent work they motivated are the subject of this paper.

The starting point for this study of the differential topology of complex algebraic surfaces is the theory of connections on certain auxiliary principal $G$-bundles over 4-manifolds, where $G$ is a compact Lie group. Let $M$ be a closed oriented $C^{\infty}$-manifold, $P \stackrel{\pi}{\rightarrow} M$ a principal $G$-bundle, and ad $P$ the bundle associated to $P$ via the adjoint representation of $G$ on its Lie algebra. We denote by $\Omega^{i}(M)$ the $C^{\infty} i$-forms on $M$ and by $\Omega^{i}(M ;$ ad $P)$ the $C^{\infty}$ $i$-forms on $M$ with values in ad $P$. If $A$ is a connection on $P$, then its curvature $F_{A}$ lies in $\Omega^{2}(M ; \operatorname{ad} P)$. Given a Riemannian metric $g$ on $M$, there are induced Hodge $*$-operators

$$
\Omega^{i}(M) \stackrel{*}{\rightarrow} \Omega^{n-i}(M) \quad \text { and } \quad \Omega^{i}(M ; \operatorname{ad} P) \stackrel{*}{\rightarrow} \Omega^{n-i}(M ; \operatorname{ad} P)
$$

where $n=\operatorname{dim} M$. If $n=4$, then both $* F_{A}$ and $F_{A}$ belong to $\Omega^{2}(M ; \operatorname{ad} P)$. Moreover, there is a natural energy functional on the space of all connections: the Yang-Mills functional

$$
A \mapsto \int_{M}\left\|F_{A}\right\|^{2} d \text { vol. }
$$

The Euler-Lagrange equations for critical points (i.e., the Yang-Mills equations) are

$$
\left\{\begin{array}{c}
D_{A} F_{A}=0 \\
D_{A} * F_{A}=0
\end{array}\right\}
$$

where $D_{A}$ is the covariant derivative on $\Omega^{2}(M ; \operatorname{ad} P)$ associated to the connection $A$. The solutions to these equations are the Yang-Mills connections. (Notice that $D_{A} F_{A}=0$ is the Bianchi identity which holds for all connections.) The natural group of symmetries of $P, \mathcal{G}(P)=\{\varphi: P \rightarrow P \mid \pi \circ \varphi=\pi$ and $\varphi(p \cdot g)=\varphi(p) \cdot g$ for all $p \in P$ and $g \in G\}$, is called the gauge group. It 
acts on the space of connections leaving invariant the Yang-Mills functional. Thus, it leaves invariant the subspace of Yang-Mills connections.

There is a special subspace of Yang-Mills connections to which we often restrict. These are the anti-self-dual connections (resp. self-dual connections) which by definition are connections $A$ whose curvature satisfies

$$
F_{A}=-* F_{A} \quad\left(\text { resp. } F_{A}=* F_{A}\right) \text {. }
$$

(Of course, self-dual and anti-self-dual connections are Yang-Mills connections.) In the special case that $G=\mathrm{SU}(2)$, the anti-self-dual connections (resp. self-dual connections) are the absolute minima for the Yang-Mills functional provided $c_{2}(P)>0$ (resp. $\left.c_{2}(P)<0\right)$.

Let us illustrate all of this with the abelian case, that is, the case of a U(1)-bundle $P$ over a simply connected 4-manifold $M$. Since U(1) is abelian, ad $P$ is a trivial $\mathbf{R}$-bundle. Hence, the curvature of a connection $A$ on $P$ is an ordinary 2-form $F_{A} \in \Omega^{2}(M ; \mathbf{R})$. Furthermore, $D_{A}$ is ordinary exterior derivative. Thus, the Bianchi identity simply says that $F_{A}$ is closed. Of course, its cohomology class is $c_{1}(P) \in H^{2}(M ; \mathbf{R})$. The Yang-Mills equations say that $F_{A}$ is also closed, i.e., harmonic. The existence of such connections is completely understood: By Hodge theory every cohomology class has a unique harmonic representative. Furthermore, given a closed form $F$ representing $c_{1}(P)$, there is a connection $A$ on $P$ with $F_{A}=F$. Thus, every U(1)-bundle $P$ has Yang-Mills connections and the curvature of any such connection is the unique harmonic representative for $c_{1}(P)$. The nonuniqueness of these YangMills connections is accounted for by the gauge group. Since $M$ is simply connected, any two connections with the same curvature lie in the same $\mathcal{G}(P)$ orbit. Hence, the set of all Yang-Mills connections on the U(1)-bundle $P$ are all equivalent by the gauge group.

Maxwell's equations for electromagnetism in the vacuum can be interpreted as the Yang-Mills equations on a U(1)-bundle over space-time. More explicitly, let $B(x, y, z, t)=\left(B_{x}, B_{y}, B_{z}\right)$ and $E(x, y, z, t)=\left(E_{x}, E_{y}, E_{z}\right)$ be the magnetic and electric fields. Then consider the 2 -form over $(x, y, z, t)$-space

$$
\begin{aligned}
\Omega= & E_{x} d x \wedge d t+E_{y} d y \wedge d t+E_{z} d z \wedge d t \\
& +B_{x} d y \wedge d z+B_{y} d z \wedge d x+B_{z} d x \wedge d y
\end{aligned}
$$

The equations $\operatorname{div} B=0$ and $\nabla \times E=-\partial B / \partial t$ say that $d \Omega=0$. The equations $\operatorname{div} E=0$ and $\nabla \times B=\partial E / \partial t$ say that $d(* \Omega)=0$ for the Hodge *-operator associated to the metric

$$
d s^{2}=d x^{2}+d y^{2}+d z^{2}-d t^{2} .
$$

Thus, Maxwell's equations can be formulated as saying that $\Omega$ is a harmonic 2-form. Let $A=\left(A_{x}, A_{y}, A_{z}\right)$ be the vector potential and $\varphi$ be the scalar potential, and set

$$
\omega=A_{x} d x+A_{y} d y+A_{z} d z-\varphi d t .
$$

The equations $E=-\nabla \varphi-\partial A / \partial t$ and $B=\nabla \times A$ say that $\omega$ is a 1 -form satisfying $d \omega=\Omega$. It is natural to view $\omega$ as a connection 1-form on a 
$\mathrm{U}(1)$-bundle over space-time with curvature $\Omega$. The reason is that changing the potentials $(A, \varphi)$ by $(\nabla \psi,-\partial \psi / \partial t)$ corresponds to acting on $\omega$ by the gauge group. This then is the formulation of electromagnetism in terms of Yang-Mills connections on U(1)-bundles over space-time.

The above discussion shows that Yang-Mills theory for $G=\mathrm{U}(1)$ essentially reduces to Hodge theory. Of course Hodge theory is a powerful tool for the study of the topology of smooth algebraic varieties. Still it is natural to ask if more information is available when we take $G$ to be nonabelian. In this case the Yang-Mills equations or the anti-self-dual equations are nonlinear PDEs. For this reason, we expect the theory to be both more subtle and richer. The choice of the group $G$ will be dictated by physical and/or mathematical considerations. Thus, $\mathrm{SU}(2) \times \mathrm{U}(1)$ arises in attempting to unify the weak force with the electromagnetic force [44], and $\mathrm{SU}(3)$ arises for the strong force [18]. Although one can develop the mathematical formalism for any compact Lie group, the details are best understood in the cases $G=\mathrm{SU}(2)$ and $G=\mathrm{SO}(3)$. However, for reasons that will become apparent below, we shall confine ourselves to $G=\mathrm{SU}(2)$.

We fix now a smooth 4-manifold $M$ with Riemannian metric $g$ and a principal SU(2)-bundle $P$ over $M$ with $c_{2}(P)>0$. The recent breakthrough in understanding the differential topology of algebraic surfaces comes from considering the moduli space $\mathcal{M}(P, g)$ of all anti-self-dual connections on $P$ modulo the gauge group of the bundle. The basic analytic properties of $\mathcal{M}(P, g)$ have been established by Uhlenbeck [42], Taubes [38], and Donaldson $[\mathbf{1 1}, \mathbf{1 3}$ ] (cf. also Atiyah-Hitchin-Singer [3]), and the method, which we shall describe, of applying these results to $C^{\infty} 4$-manifolds was pioneered by Donaldson. For a generic metric, it turns out that $\mathcal{M}(P, g)$ is a finite-dimensional orientable submanifold of $X(P)$, the space of all connections modulo the gauge group. Its dimension $d$ can be computed from the Atiyah-Singer index theorem. The basic idea is to try to use $\mathcal{M}(P, g)$ to define a cycle class in the homology of $X(P)$. Unfortunately, $\mathcal{M}(P, g)$ is rarely compact. Thus, one is faced with the problem of trying to compactify it in a canonical way. This is one of the main technical points in the theory. But this problem can be surmounted, at least for $c_{2}(P)$ sufficiently large, and one obtains a homology class $[\mathcal{M}(P, g)]$ in $H_{*}(\mathcal{X}(P))$. Of course the space $\mathcal{M}(P, g)$ depends on the Riemannian metric $g$. However, as we vary $g, \mathcal{M}(P, g)$ usually varies by a homology in $\chi(P)$, and in the exceptional cases its failure to do so can be precisely described. Finally, there is an explicit description of $H^{*}(\chi(P) ; \mathbf{Q})$ in terms of $H_{*}(M)$. In particular, using the second Chern class of the universal bundle on $M \times \chi(P)$ one can identify $H_{2}(M)$ with $H^{2}(X(P))$. Furthermore, the product in cohomology induces an injection of the polynomial algebra on $H^{2}(X(P))$ into $H^{2 *}(\chi(P))$. Thus, evaluating elements of $H^{d}(\mathcal{X}(P))$ on $[\mathcal{M}(P, g)]$ gives a polynomial of degree $d / 2$ on $H^{2}(M)$ which is a differential invariant of $M$.

This machinery is especially well suited to studying algebraic surfaces. The reason traces back to a theorem of Donaldson [12]: When $M$ is an algebraic surface and $g$ is an appropriate Kähler metric one can identify $\mathcal{M}(P, g)$ with the moduli space of stable holomorphic bundle structures on the complex 2-plane bundle associated to $P$. (It is for this reason that we consider the 
special unitary group as opposed to the special orthogonal group.) Consequently, the moduli spaces of stable bundles over an algebraic surface, which are algebro-geometric in nature, give important $C^{\infty}$ invariants of the underlying smooth manifold. Quite independently of these considerations, algebraic geometers have been studying holomorphic rank-2 vector bundles over algebraic surfaces for twenty-five years. They have developed a wealth of techniques for classifying them and studying their moduli spaces.

Since the inception of the theory of 4-manifolds, a guiding question has been:

What is the relationship between smooth 4-manifold theory and the theory of algebraic surfaces?

For the reasons indicated above, the methods of gauge theory give us powerful new tools to attack this question. Of course, one doesn't expect a complete correspondence. For example, any finitely presented group occurs as the fundamental group of a closed orientable smooth 4-manifold, but fundamental groups of algebraic surfaces are severely restricted. One such restriction is given by Hodge theory [19]: $b_{1} \equiv 0(\bmod 2)$ for an algebraic surface. For more subtle restrictions see [9]. These examples suggest that one should first focus attention on the simply connected case. In this paper, we shall consider the $C^{\infty}$-topology of complex algebraic surfaces, state some results and give some conjectures and speculation which these results suggest (at least to us).

1. A brief review of the homotopy theory of 4-manifolds. Throughout this section we are concerned with simply connected, closed, oriented, smooth 4-manifolds. Any such manifold $M$ has an orientation class $[M] \in$ $H_{4}(M ; Z)$. Cap product with it induces the Poincaré duality isomorphism

$$
\bigcap[M]: H^{i}(M ; \mathbf{Z}) \stackrel{\cong}{\longrightarrow} H_{4-i}(M ; \mathbf{Z}) \text {. }
$$

Since $M$ is simply connected, $H_{1}(M ; Z)=0$. By Poincaré duality it follows that $H_{3}(M ; \mathbf{Z})=0$ and that $H_{2}(M ; \mathbf{Z})$ is a free abelian group of finite rank. Furthermore, Poincaré duality induces an intersection pairing

$$
q_{M}: H_{2}(M ; \mathbf{Z}) \otimes H_{2}(M ; \mathbf{Z}) \rightarrow \mathbf{Z}
$$

which is symmetric and nonsingular, i.e., unimodular. (We shall also use the dual pairing on cohomology which is given by the cup product pairing and evaluation on the top class.) This then leads to the first invariant associated to a 4-manifold: the algebraic isomorphism class of the pairing $\left(H_{2}(M ; \mathbf{Z}), q_{M}\right)$.

The isomorphism classification of such pairings is more or less understood: Let $(A, q)$ be a unimodular, symmetric pairing. Its rank is the rank of the free abelian group $A$. Its index $I(q)$ is defined as follows: Over $\mathbf{R}$ the pairing can be diagonalized, i.e., there is an $\mathbf{R}$-basis $x_{1}, \ldots, x_{k}$ for $A \otimes \mathbf{R}$ such that $q\left(x_{i}, x_{j}\right)=$ 0 for $i \neq j$. We set $p(q)$ equal to the number of $x_{i}$ for which $q\left(x_{i}, x_{i}\right)>0$. Similarly, we define $n(q)$ as the number of $x_{i}$ for which $q\left(x_{i}, x_{i}\right)<0$. Since $q$ is nonsingular $p(q)+n(q)$ is equal to the rank. We define $I(q)=p(q)-n(q)$. We say that a pairing is positive (resp. negative) definite if $n(q)=0$ (resp. $p(q)=0)$. Lastly, we say that $q$ is even if $q(a, a) \equiv 0(\bmod 2)$ for all $a \in A$. Otherwise we say the pairing is odd. This defines the parity of $q$. 
The basic result in the classification of unimodular pairings (see [36]) says:

(1) if $(A, q)$ is indefinite it is determined up to isomorphism by its rank, index and parity; and

(2) there are only finitely many definite pairings of a given rank.

There are some relationships among these invariants. Besides the obvious ones, $|I(q)| \leq \operatorname{rank} A$ and $I(q) \equiv \operatorname{rank}(A)(\bmod 2)$, there is one more: If $q$ is even then $I(q) \equiv 0(\bmod 8)$. Subject to these restrictions, all values of the rank, index and parity are possible.

Returning to the 4-manifold, we set $b_{2}^{+}(M)=p\left(q_{M}\right), b_{2}^{-}(M)=n\left(q_{M}\right)$, and $I(M)=I\left(q_{M}\right)$. We call $I(M)$ the index of $M$. It turns out that two simply connected 4-manifolds $M$ and $N$ are homotopy equivalent if and only if their pairings $q_{M}$ and $q_{N}$ are isomorphic, see [45] and [31]. Similarly the group of homotopy automorphisms of $M$ is commensurate to the group of automorphisms of the intersection pairing.

On the level of homotopy types every pairing is realized. That is to say, given an algebraic pairing $(A, q)$ there is a simply connected complex $X$ satisfying Poincaré duality of dimension 4 with $\left(H_{2}(X ; \mathbf{Z}), q_{X}\right)$ isomorphic to $(A, q)$. Such an $X$ is unique up to homotopy equivalence.

Freedman [16] recently determined the homeomorphism classification of simply connected 4-manifolds. He showed that every pairing occurs up to isomorphism as the intersection pairing of a simply connected topological 4-manifold. If the pairing is even then all manifolds realizing it are homeomorphic. If the pairing is odd, then there are exactly two homeomorphism classes of manifolds realizing it. Exactly one of these is stably smoothable (i.e., is such that the product with $\mathbf{R}$ carries a smooth structure).

Before Donaldson's recent results the only known restriction on forms realized by smooth manifolds was Rokhlin's theorem [35]: If the intersection pairing of a smooth 4-manifold $M$ is even then $I(M) \equiv 0(\bmod 16)$. Nothing was known about how many differentiably distinct manifolds represent a given pairing. (The case of the trivial pairing is the 4-dimensional smooth Poincaré Conjecture). The landscape changed drastically about three or four years ago when Donaldson introduced the techniques that we have briefly described above. We shall mention some of his results in what follows, especially in §4.

2. A brief review of the classification of algebraic surfaces. For simplicity, we shall concentrate on simply connected algebraic surfaces, referring the reader to [5 or $\mathbf{1 9}$ ] for a more complete description of the non-simply connected case. Let $X$ be a (smooth) algebraic surface. We begin by describing the plurigenera $P_{n}(X)$ and the Kodaira dimension $\kappa(X)$. Let $K_{X}$ be the canonical line bundle of $X$. Its local holomorphic sections are holomorphic 2-forms on $X$, i.e., in local coordinates $\left\{z_{1}, z_{2}\right\}$, a local section of $K_{X}$ is of the form $f\left(z_{1}, z_{2}\right) d z_{1} \wedge d z_{2}$, where $f\left(z_{1}, z_{2}\right)$ is a holomorphic function. For $n>0$, let $K_{X}^{\otimes n}$ be the $n$th tensor power of $K_{X}$. It is a line bundle whose local sections look like $g\left(z_{1}, z_{2}\right)\left(d z_{1} \wedge d z_{2}\right)^{n}$. Set $P_{n}(X)=n$th plurigenus $=\operatorname{dim}_{\mathrm{C}} H^{0}\left(X, K_{X}^{\otimes n}\right)$. The geometric genus of $X$ is defined to be $p_{g}(X)=P_{1}(X)$; it is the number of linearly independent holomorphic 2-forms 
on $X$. By the Hodge index theorem, $b_{2}^{+}(X)=2 p_{g}(X)+1$, and thus $p_{g}(X)$ is a homotopy invariant of $X$.

If $P_{n}(X)=0$ for all $n>0$, we set $\kappa(X)=-\infty$. Otherwise, $P_{n}(X)=O\left(n^{a}\right)$ for some integer $a, 0 \leq a \leq 2$ (see [5]), and we let the smallest such $a$ be $\kappa(X)$, the Kodaira dimension of $X$. Thus, in general, $\kappa(X) \in\{-\infty, 0,1,2\}$.

We also need to recall the notion of blowing up an algebraic surface $X$. Given the surface $X$ and a point $p \in X$, there is an algebraic surface $\tilde{X}$, a holomorphic map $\rho: \tilde{X} \rightarrow X$ and a holomorphic curve $E \cong \mathbf{C} P^{1} \subseteq \tilde{X}$ such that $\rho$ induces an isomorphism $\tilde{X}-E \rightarrow X-\{p\}$ and $\rho(E)=\{p\}$. Moreover, if $[E]$ denotes the cohomology class Poincaré dual to $E$, then the self-intersection number $[E] \cdot[E]$ of $E$ on $\tilde{X}$ is -1 . This process, which is uniquely specified by $X$ and $p$, is called blowing up $X$ at $p$. We have $K_{\tilde{X}}=\rho^{*} K_{X}+E$ (as divisor classes on $\tilde{X})$. From this it is easy to see that $p_{g}(X), P_{n}(X)$, and $\kappa(X)$ are not affected by blowing up a point of $X$. Topologically, $\tilde{X}$ is diffeomorphic (in an orientation-preserving way) to $X \# \overline{\mathbf{C P}}^{2}$, where $\overline{\mathbf{C P}}^{2}$ is $\mathbf{C} P^{2}$ with the opposite orientation to the one induced by the complex structure. We may invert the procedure: if $\tilde{X}$ is an algebraic surface and $E$ a holomorphic curve in $\tilde{X}$ with $E \cong \mathbf{C} P^{1}$ and $[E] \cdot[E]=-1$, then there exists $\rho: \tilde{X} \rightarrow X$ and $\rho(E)=p \in X$ such that $\tilde{X}$ is the blow-up of $X$ at $p$. Such a curve $E$ is called an exceptional curve. We say that $X$ is obtained from $\tilde{X}$ by blowing down $E$. An algebraic surface is said to be minimal if it has no exceptional curve, i.e., if it cannot be blown down. Every algebraic surface can be blown down to a (not necessarily unique) minimal surface.

With this said, a simply connected algebraic surface $X$ of Kodaira dimension $-\infty$ can be described as follows. It is a rational surface, which means that $X$ may be obtained from $\mathbf{C} P^{2}$ by iterating the procedure of blowing up finitely many times, followed by iterating the procedure of blowing down finitely many times. Equivalently, the field of meromorphic (or rational) functions on $X$ is isomorphic to $\mathbf{C}\left(x_{1}, x_{2}\right)$. The diffeomorphism types of $X$ are easy to describe: such an $X$ is diffeomorphic either to $\mathbf{C} P^{2} \# n \overline{\mathbf{C P}}^{2}, n \geq 0$, or to $S^{2} \times S^{2}$. Finally, we should mention the famous Castelnuovo criterion: an algebraic surface $X$ is rational if and only if it is simply connected and $P_{2}(X)=0$. (Actually, it suffices to assume that $b_{1}(X)=P_{2}(X)=0$.) A non-simply connected surface $S$ with $\kappa(S)=-\infty$ is a ruled surface. More precisely, a minimal ruled surface is an algebraic surface $S$, together with a holomorphic map $\pi: S \rightarrow C$, where $C$ is an algebraic curve, such that all fibers $\pi^{-1}(x), x \in C$, are isomorphic to $\mathbf{C} P^{1}$. A general ruled surface $\tilde{S}$ is then a blow-up of a minimal ruled surface $S$, and thus there is an induced map $\tilde{S} \rightarrow C$. If $g(C)=0$, then $S$ and $\tilde{S}$ are rational. Otherwise, the map $\tilde{S} \rightarrow C$ is unique, although the blow-down $\tilde{S} \rightarrow S$ is not. (The nonuniqueness is exactly accounted for by the so-called elementary transformations $[22, \mathrm{p}$. 416].)

If $S$ is an algebraic surface with $\kappa(S) \geq 0$, then $S$ can be uniquely blown down to a minimal surface $S_{\min }$ which is called its minimal model. The uniqueness implies that there are a finite number of marked curves on $S$, the 
curve components of the fibers of $S$ over $S_{\min }$. These are the only curves which can be contracted under any degree one holomorphic map of $S$ to another smooth surface. (By contrast, it is possible for a rational surface to have infinitely many exceptional curves [22, p. 409].) Thus, the classification of surfaces with $\kappa \geq 0$ is reduced to the study of their minimal models.

Let us consider minimal surfaces $S$ with $\kappa(S)=0$. If $S$ is simply connected, then $S$ is a $K 3$ surface. Such surfaces are parametrized by an (extensively studied) irreducible 20-dimensional complex space, and consequently, all such surfaces are diffeomorphic. If $S$ is not simply connected, then it is a quotient of a $K 3$ surface or a complex torus by a finite group acting freely. Moreover, such surfaces are parametrized by a finite number of connected complex spaces (in fact, the list is very short; cf. [5 or 19]). From this discussion, it follows that minimal surfaces $S$ with $\kappa(S)=0$ represent only finitely many $C^{\infty}$-types of 4-manifolds.

If $S$ is a minimal surface with $\kappa(S)=1$, then $S$ is elliptic, i.e. there is a canonically defined holomorphic map $\pi: S \rightarrow C$, where $C$ is an algebraic curve, such that $\pi^{-1}(t)$ is a smooth algebraic curve of genus one (i.e., an elliptic curve), for almost all $t \in C$. Such a fibration may have multiple fibers [24]. The surface can always be deformed until the multiple fibers are smooth. In local analytic coordinates near a multiple fiber, $\pi: S \rightarrow C$ is then given by $\pi(z, t)=t^{m}$ where $m>1$ is the multiplicity. If $\pi_{1}(S)=0$, then $C \cong \mathbf{C} P^{1}$. Furthermore, there are at most two multiple fibers and the multiplicities are relatively prime. The simply connected elliptic surfaces without multiple fibers fall into countably many connected, in fact irreducible, families indexed by $p_{g}$ (or equivalently by $b_{2}^{+}$). Any elliptic surface with multiple fibers is obtained from one without multiple fibers by log transforms [25]. From this one can show that simply connected elliptic surfaces are divided into countably many irreducible families indexed by $p_{g}, p$, and $q$ where $p, q \geq 1$ are the multiplicities and are relatively prime. But if $p_{g}=0$ and at least one of $p$ and $q$ is 1 the resulting surfaces are rational. If $p_{g}=1$ and $p=q=1$ then the resulting surface is a $K 3$ surface. In all other cases, we get a surface of Kodaira dimension one.

Finally, if $\kappa(S)=2$, then $S$ is of general type, which for our purpose will be simply synonymous with "other." For example, a smooth hypersurface of degree at least 5 in $\mathbf{C P} P^{3}$ is of general type. More generally, we define a complete intersection surface $S \subseteq \mathbf{C} P^{N}$ to be the transverse intersection of $(N-2)$ smooth hypersurfaces of degrees $d_{1}, \ldots, d_{N-2}$. With very few exceptions, these are all of general type. Surfaces of general type remain a vast and uncharted wilderness, whose study (largely consisting of constructing examples), goes by the name of "geography". Indeed, the name general type reflects the feeling that, given the bewildering array of examples of surfaces of general type, most surfaces fall into this class. In spite of this, from the point of view of classifying smooth 4-manifolds, one can at least say the following. If we fix the homotopy type of a 4-manifold, all but finitely many diffeomorphism types of algebraic surfaces of the given homotopy type will be (possibly nonminimal) surfaces with $\kappa=1$ (i.e. blown up elliptic surfaces). Put slightly differently, the (possibly nonminimal) surfaces of general type of 
a given homotopy type fall into at most finitely many irreducible families. This will be discussed in more detail in the remarks after Conjecture 1 in the next section. We summarize the situation in the Table.

\section{TABLE}

\section{Classification of algebraic surfaces}

\begin{tabular}{|c|c|c|c|}
\hline$\kappa$ & & $\begin{array}{l}\text { simply connected } \\
\text { case }\end{array}$ & $\begin{array}{l}\text { non-simply connected } \\
\text { case }\end{array}$ \\
\hline$-\infty$ & \multicolumn{2}{|c|}{$\begin{array}{l}\text { rational; diffeomorphic } \\
\text { to } S^{2} \times S^{2} \text { or } \mathbf{C} P^{2} \# n \overline{\mathbf{C} P}{ }^{2}\end{array}$} & $\begin{array}{l}\text { ruled over a curve of } \\
\text { genus at least one }\end{array}$ \\
\hline 0 & \multirow{3}{*}{$\begin{array}{c}\text { existence } \\
\text { of a } \\
\text { unique } \\
\text { minimal } \\
\text { model }\end{array}$} & $S_{\min }$ is a $K 3$ surface & $\begin{array}{l}S_{\min } \text { is a complex torus } \\
\text { an Enriques surface, or } \\
\text { a hyperelliptic surface }\end{array}$ \\
\hline 1 & & $\begin{array}{l}S_{\min } \text { is an elliptic } \\
\text { surface, described by } \\
p_{g}, p, q \geq 1\end{array}$ & $\begin{array}{l}S_{\min } \text { is a more general } \\
\text { elliptic surface }\end{array}$ \\
\hline 2 & & general type & general type \\
\hline
\end{tabular}

Simply connected elliptic surfaces $X$ with $p_{g}(X)=0$ occupy a special case in the classification. We will call a minimal such surface a Dolgachev surface; its $C^{\infty}$-type is determined by $p, q \in \mathrm{N}, p, q \geq 2$ and g.c.d. $(p, q)=1$. Historically, their interest was as follows. Castelnuovo proved in 1896 that $X$ is rational if and only if $b_{1}(X)=P_{2}(X)=0$. This result is akin to the well-known fact that a compact Riemann surface $C$ is $\mathbf{C} P^{1}$ if and only if the genus $g(C)$ is 0 . However, it is not clear that $P_{2}(X)$ is a topological invariant. As we shall see, $P_{2}(X)$ is not determined by the homotopy type of $X$. A more natural invariant, which does depend only on the homotopy type, would be $p_{g}(X)=P_{1}(X)$. However, Enriques constructed a surface $S$ (which now bears his name) with $p_{g}(S)=b_{1}(S)=0$ which is not rational. For the Enriques surface $S$, we have $\kappa(S)=0$ and $\pi_{1}(S)=\mathbf{Z} / 2 \mathrm{Z}$; the universal cover of $S$ is a $K 3$ surface. Later, Godeaux constructed a surface $T$ of general type with $p_{g}(T)=b_{1}(T)=0$, and $\pi_{1}(T)=\mathbf{Z} / 5 \mathrm{Z}$. Severi posed the problem of whether a surface $S$ with $H_{1}(S ; \mathbf{Z})=0$ and $p_{g}(S)=0$ was necessarily rational. Dolgachev constructed his counterexamples in 1966 [10]; they are in fact simply connected. Only in 1982 did Barlow [4] produce a simply connected surface of general type with $p_{g}=0$. It is unknown whether Barlow's surface is diffeomorphic to a rational surface (this seems quite unlikely), and it is known that the Dolgachev surfaces and their blow-ups are never diffeomorphic to rational surfaces [17]. (We shall discuss this further in the next section.) As these examples show, $P_{2}(X)$ is not a homotopy invariant, and indeed by Freedman's result [16], it is not a homeomorphism invariant. One can ask if $P_{2}(X)$, or more generally $P_{n}(X)$, is a diffeomorphism invariant. This is unknown at present.

3. Some conjectures. The first application of gauge theory to the differential topology of algebraic surfaces was Donaldson's example showing that the $h$-cobordism theorem does not extend to dimension 4 [14]. He showed that 
a Dolgachev surface with multiple fibers of orders 2 and 3 is not diffeomorphic to $\mathbf{C} P^{2}$ blown up at 9 points, although by Dolgachev's work the surfaces are homotopy equivalent and hence $h$-cobordant $[10]$. By Freedman's result [16] this amounts to putting two nondiffeomorphic smooth structures on the topological manifold $\mathbf{C} P^{2} \# 9 \overline{\mathbf{C P}}^{2}$. Donaldson's example was generalized in [17] to Dolgachev surfaces and their blow-ups and independently in [33] to Dolgachev surfaces.

One consequence of $[\mathbf{1 7}]$ is a proof of the existence of infinitely many nondiffeomorphic smooth structures on $\mathbf{C} P^{2} \# n \overline{\mathbf{C P}}^{2}$ for all $n \geq 9$. This result is qualitatively very different from results in higher dimensions [37], which say roughly that a smooth simply connected manifold of dimension $\geq 5$ is determined up to finitely many diffeomorphism possibilities by its homotopy type and Pontrjagin classes. Another consequence of [17] is that for all blown up Dolgachev surfaces, the automorphisms of $\left(H^{2}(S), q_{S}\right)$ which can be realized by self-diffeomorphisms satisfy nontrivial conditions. These will be explained in more detail below, but to mention a qualitative result here, the subgroup of such automorphisms has infinite index in the full group of all automorphisms of the form. Once again this is qualitatively very different from the results in higher dimensions (cf. [37]).

It is our feeling that these results for Dolgachev surfaces are but the tip of the iceberg, and that the techniques used to study them have wide applications. The conjectures we make here are natural generalizations of what is now known in the case of Dolgachev surfaces.

DEFINITION. Two smooth complex manifolds $Z_{1}$ and $Z_{2}$ are of the same deformation type (also called c-homotopic in the older literature) if there exist connected complex spaces $X$ and $T$, a smooth proper holomorphic map $\Phi: X \rightarrow T$, and points $t_{1}, t_{2} \in T$ with $Z_{i} \cong \Phi^{-1}\left(t_{i}\right)$.

Equivalently, deformation type is the equivalence relation generated by declaring that two complex manifolds are equivalent if they are both fibers in a proper smooth map between two connected complex manifolds. If $Z_{1}$ and $Z_{2}$ are of the same deformation type, their underlying manifolds are diffeomorphic, via a diffeomorphism which preserves the canonical orientations and the canonical classes.

CONJECTURE 1. The natural map

$$
\left\{\begin{array}{c}
\text { algebraic surfaces } \\
\text { modulo deformation } \\
\text { type }
\end{array}\right\} \rightarrow\left\{\begin{array}{c}
\text { oriented smooth 4-manifolds } \\
\text { modulo orientation-preserving } \\
\text { diffeomorphisms }
\end{array}\right\}
$$

is finite-to-one.

REMARKS. (1) In the definition of deformation type we do not require that all fibers of the map $\Phi: X \rightarrow T$ be algebraic, merely that they be complex surfaces. However, it can be shown that requiring all fibers to be algebraic gives an equivalent conjecture, although a more natural category to work in is that of Kähler surfaces.

(2) It suffices to prove Conjecture 1 for blown up elliptic surfaces. The reason is that the result holds if we restrict to the category of algebraic surfaces 
which have nonelliptic minimal models. This fact is more or less well known. (See $\left[17\right.$, III.3.4] for the case $p_{g}=0$.) We have divided surfaces into those of Kodaira dimension $-\infty, 0,1,2$ and we are claiming that the result holds for surfaces with $\kappa \neq 1$. It clearly suffices to consider the cases $\kappa=-\infty$, $\kappa=0$, and $\kappa=2$ independently. For $\kappa=-\infty$, a straightforward argument with elementary transformations shows that, for a possibly nonminimal ruled surface, deformation type and diffeomorphism type coincide. The surfaces of Kodaira dimension zero (possibly non-simply connected) fall into a very short finite list of $C^{\infty}$-types, and here again deformation type and diffeomorphism coincide. Lastly, we consider (possibly nonminimal) surfaces $Y$ of general type. The Noether formula says

$$
c_{1}^{2}(Y)+c_{2}(Y)=12 \chi\left(O_{Y}\right) .
$$

From the Hirzebruch Index Theorem one derives that $c_{1}^{2}(Y)=3 I(Y)+2 \chi(Y)$. Thus $c_{1}^{2}\left(Y_{\min }\right)$ is bounded solely in terms of the homotopy type of $Y$, as $c_{1}^{2}\left(Y_{\min }\right) \leq c_{1}^{2}(Y)+b_{2}(Y)$. By results of Moishezon, Kodaira, and Bombieri (with the sharpest bounds due to Bombieri [8]), minimal surfaces with $c_{1}^{2}$ less than or equal to a given bound may all be mapped birationally to a fixed projective space $\mathbf{C} P^{N}$. According to the general properties of the Hilbert scheme, such surfaces may then be parametrized by a finite union of quasiprojective varieties, and the same will be true after a bounded number of blow-ups. Hence, the number of deformation types of surfaces of general type in a given homotopy type, and thus the number of diffeomorphism types of such surfaces, is finite.

In [17], we proved Conjecture 1 for Dolgachev surfaces and their blow-ups. This established Conjecture 1 for all simply connected algebraic surfaces with $p_{g}=0$ (or equivalently with $b_{2}^{+}=1$ ). Lübke and Okonek [27] and F. Maier [28] have verified Conjecture 1 for minimal elliptic surfaces with $p_{g}=0$ and finite cyclic fundamental group.

(3) Surprisingly, if Conjecture 1 holds for simply connected surfaces, then it holds for all surfaces. First, one reduces to the case of (possibly blown up) elliptic surfaces, by the above remark. Next, if an elliptic surface has a finite fundamental group, its universal cover is again elliptic, and a straightforward argument reduces this case to the simply connected case. Finally, if the fundamental group is infinite, one associates to the surface a certain 2-dimensional orbifold $V$, which consists of the base $C$ of the elliptic fibration $\pi: S \rightarrow C$, together with points $x_{1}, \ldots, x_{k} \in C$ and multiplicities $p_{1}, \ldots, p_{k}$ at $x_{1}, \ldots, x_{k}$. Here, the $x_{i}$ are the images of the multiple fibers and the $p_{i}$ are the multiplicities of the corresponding fibers. One can show that the fundamental group of $S$ determines the "orbifold fundamental group" of $V$. By a result in surface topology, this orbifold fundamental group determines $V$ and hence $g(C)$ and the $p_{i}$, unless $g(C)=0$ and $k \leq 2$. However, in this last case, either the fundamental group of $S$ is finite (a case which we have already dealt with), or it is infinite and this case can be handled by Kodaira's work on elliptic surfaces of type VII [25]. Compare also Ue [41], who discusses the relationship between the fundamental group and the diffeomorphism classification for the case of infinite fundamental group. 
An analogous conjecture is false for complex dimension $>2$. For example, let $S(p, q)$ be a Dolgachev surface with multiple fibers of orders $p$ and $q$ such that g.c.d. $(p, q)=1$. The 8-manifolds $S(p, q) \times S(p, q)$, as $(p, q)$ ranges over unordered pairs of relatively prime natural numbers, are all of the same homotopy type and have the same Pontrjagin classes. One can show that there are at most two possibilities for the diffeomorphism type. Hence, for infinitely many choices of $p$ and $q$, the $S(p, q) \times S(p, q)$ are all diffeomorphic. However, the order of divisibility of the canonical line bundle becomes arbitrarily large as $p, q \rightarrow \infty$, and this easily implies that the $S(p, q) \times S(p, q)$ cannot all fall into finitely many deformation types.

On the positive side, however, Kollár has shown, by a simple but clever argument, the following. Let $M^{2 n}$ be a smooth oriented $2 n$-manifold with $b_{2}(M)=1$. Then the set of deformation types of Kähler (or equivalently algebraic) complex structures on $M$ is finite.

SPECULATION. Is it possible that the map in Conjecture 1 is actually one-to-one?

No counterexamples to this speculation are known, even for non-simply connected surfaces. In this connection, the case of elliptic surfaces with infinite fundamental group seems very accessible. As further evidence, Moishezon has found many examples of pairs of simply connected surfaces of general type which can be shown to be orientation-preserving homeomorphic but not diffeomorphic, and hence not of the same deformation type. On the other hand, establishing the above speculation for all surfaces of general type seems to be extremely difficult.

It seems to be the case that the nature of the differential topology of a surface depends on its Kodaira dimension, with the case $\kappa=-\infty$ being qualitatively different. Thus, our next conjectures are for surfaces with $\kappa \geq 0$.

CONJECTURE 2. Let $S$ be an algebraic surface with $\kappa(S) \geq 0$, let $S_{\min }$ be the minimal model and let $\rho: S \rightarrow S_{\min }$ be the natural map. If $f: S \rightarrow S$ is an orientation-preserving diffeomorphism, then $f^{*}$ preserves the subspace $\rho^{*} H^{2}\left(S_{\min } ; \mathbf{Z}\right) \subseteq H^{2}(S ; \mathbf{Z})$.

If $E_{1}, \ldots, E_{k}$ are the exceptional fibers of $\rho$ (assuming that we have blown up $k$ distinct points for simplicity), then $\rho^{*} H^{2}\left(S_{\min } ; \mathbf{Z}\right)^{\perp}$ is the $\mathbf{Z}$-span of $\left[E_{1}\right], \ldots,\left[E_{k}\right]$, where $\left[E_{i}\right]$ is the cohomology class dual to $E_{i}$. Thus $f^{*}$ preserves $\rho^{*} H^{2}\left(S_{\min } ; \mathbf{Z}\right)$ if and only if $f^{*}$ preserves

$$
\rho^{*} H^{2}\left(S_{\min } ; \mathbf{Z}\right)^{\perp}=\mathbf{Z}\left[E_{1}\right] \oplus \cdots \oplus \mathbf{Z}\left[E_{k}\right] ;
$$

since $\left[E_{i}\right]^{2}=-1$, this is true if and only if $f^{*}$ acts on the $\left[E_{i}\right]$ via sign changes and permutations.

CONJECTURE 3. With $S, S_{\min }$, and $\rho$ as in Conjecture 2, if $f: S \rightarrow S$ is an orientation-preserving diffeomorphism, then $f^{*}\left[\rho^{*} K_{S_{\min }}\right]= \pm\left[\rho^{*} K_{S_{\min }}\right]$.

For a minimal surface, of course, Conjecture 3 simply reads $f^{*}\left[K_{S}\right]=$ $\pm\left[K_{S}\right]$. However, it is not in general true that $f^{*}\left[K_{S}\right]= \pm\left[K_{S}\right]$ if $S$ is not minimal. The reason is that if $S$ has an exceptional curve $E$, then there is 
a diffeomorphism which realizes reflection in $[E]$ in cohomology. This diffeomorphism $f$ almost never satisfies $f^{*}\left[K_{S}\right]= \pm\left[K_{S}\right]$.

Conjectures 2 and 3 have been verified in [17] for Dolgachev surfaces and their blow-ups, thus giving an infinite family of interesting special cases. Using unpublished work of Donaldson, the authors have also verified Conjecture 3 for minimal (nonrational) simply connected elliptic surfaces and complete intersection surfaces of general type provided that $p_{g} \equiv 0(\bmod 2)$. This includes all hypersurfaces of degree $d$ in $\mathbf{C} P^{3}$, where $d \not \equiv 0(\bmod 4)$ and $d \geq 5$.

As a general comment, the classes of possibly blown up simply connected elliptic surfaces and of complete intersection surfaces seem to be quite feasible testing grounds for the validity of Conjectures 2 and 3. A key property of these surfaces is that their diffeomorphism groups are large. Paradoxically, this fact can be used to find nontrivial conditions on diffeomorphisms of these surfaces.

There are natural extensions of Conjectures 2 and 3 concerning diffeomorphisms between distinct algebraic surfaces.

CONJECTURE $2^{\prime}$. Let $S$ and $S^{\prime}$ be algebraic surfaces with $\kappa(S) \geq 0$ and $\kappa\left(S^{\prime}\right) \geq 0$. Let $f: S \rightarrow S^{\prime}$ be an orientation-preserving diffeomorphism. Let $\rho: S \rightarrow S_{\min }$ and $\rho^{\prime}: S^{\prime} \rightarrow S_{\min }^{\prime}$ be the minimal models. Then

$$
f^{*}\left(\left(\rho^{\prime}\right)^{*} H^{2}\left(S_{\min }^{\prime} ; \mathbf{Z}\right)\right)=\rho^{*} H^{2}\left(S_{\min } ; \mathbf{Z}\right) \text {. }
$$

CONJECTURE $3^{\prime}$. With assumptions as in Conjecture $2^{\prime}$ we have

$$
f^{*}\left(\rho^{\prime}\right)^{*}\left[K_{S_{\min }^{\prime}}\right]= \pm \rho^{*}\left[K_{S_{\min }}\right] .
$$

Clearly, these Conjectures imply Conjectures 2 and 3 . We shall see below (Proposition 4) that Conjectures 2 and 3 imply Conjecture $2^{\prime}$. Conjecture $3^{\prime}$ seems less accessible. As weak evidence for Conjecture $3^{\prime}$ we have the following: Let $S$ and $S^{\prime}$ be blown up Dolgachev surfaces and let $f: S \rightarrow S^{\prime}$ be a diffeomorphism. In [17] Conjecture $3^{\prime}$ was established for $f$ up to multiples, i.e., $f^{*}\left(\rho^{\prime}\right)^{*}\left[K_{S_{\min }^{\prime}}\right]=r(f) \cdot \rho^{*}\left[K_{S_{\min }}\right]$ for some $r(f) \in \mathbf{Q}$.

We give some consequences which show the power of these conjectures.

PROPOSITION 4. Suppose that $S$ is an algebraic surface, that $\kappa(S) \geq 0$ and that Conjectures 2 and 3 hold for $S$ and all its blow-ups. Assume, for simplicity, that $\rho: S \rightarrow S_{\min }$ is obtained by blowing up distinct points of $S_{\min }$. (This is always true after a deformation of $S$.) Let $\Sigma \subseteq S$ be a smoothly embedded 2-sphere and $\alpha \in H^{2}(S ; \mathbf{Z})$ be the cohomology class Poincaré dual to $\Sigma$. Suppose $\alpha \neq 0$. Then $\alpha^{2} \leq-1$ and if $\alpha^{2}=-1$, then $\alpha= \pm[E]$, where $E$ is an exceptional fiber of $\rho: S \rightarrow S_{\min }$.

PrOOF. First suppose that $\alpha^{2}=-1$. Let $R_{\alpha}: H^{2}(S ; \mathbf{Z}) \rightarrow H^{2}(S ; \mathbf{Z})$ be reflection about the hyperplane $\left(\alpha^{\perp}\right)$. Then by [17, III.2], $R_{\alpha}$ is induced by a self-diffeomorphism of $S$. It follows by hypothesis that $R_{\alpha}$ preserves the decomposition

$$
H^{2}(S ; \mathbf{Z})=\rho^{*} H^{2}\left(S_{\min } ; \mathbf{Z}\right) \oplus \bigoplus_{i} \mathbf{Z}\left[E_{i}\right]
$$


Thus, either $\alpha \in \rho^{*} H^{2}\left(S_{\min } ; \mathbf{Z}\right)$ or $\alpha \in \bigoplus_{i} \mathbf{Z}\left[E_{i}\right]$. First, suppose that $\alpha \in$ $\rho^{*} H^{2}\left(S_{\min } ; \mathbf{Z}\right)$. From Conjecture 3 we have $R_{\alpha}\left(\left[\rho^{*} K_{S_{\min }}\right]\right)= \pm\left[\rho^{*} K_{S_{\min }}\right]$. Thus, we see that either $\left[\rho^{*} K_{S_{\min }}\right]$ is a multiple of $\alpha$ or $\alpha \in\left[\rho^{*} K_{S_{\min }}\right]^{\perp}$. By a general result on surfaces with $\kappa \geq 0,\left[K_{S_{\min }}\right]^{2} \geq 0$, so the first case is impossible. Hence $\alpha \in\left[\rho^{*} K_{S_{\min }}\right]^{\perp}$. By the Wu formula,

$$
\left(\left[\rho^{*} K_{S_{\min }}\right]^{\perp}\right) \cap \rho^{*} H^{2}\left(S_{\min } ; \mathbf{Z}\right)=\rho^{*}\left(\left[K_{S_{\min }}\right]^{\perp}\right)
$$

is an even lattice, and hence does not contain any class of self-intersection -1 . This contradiction establishes that $\alpha \in \bigoplus_{i} \mathbf{Z}\left[E_{i}\right]$. Since $\alpha^{2}=-1, \alpha= \pm\left[E_{i}\right]$ for some $i$.

The case where $\alpha^{2} \geq 0$ is handled by blowing up $S$ at $\alpha^{2}+1$ distinct points along $\Sigma$, and reducing to the above case to obtain a contradiction.

Note. The characterization of embedded 2-spheres of self-intersection -1 easily implies Conjecture $2^{\prime}$.

There is a generalization of Proposition 4 to immersed 2-spheres.

Proposition 5. Assume that $\rho: S \rightarrow S_{\min }$ is as in Proposition 4. Let $\Sigma \rightarrow S$ be a generically immersed 2-sphere with Poincaré dual cohomology class $\alpha \neq 0$. Let $d_{+}$(resp. $d_{-}$) be the number of double points of $\Sigma$ where the local self-intersection is +1 (resp. -1$)$. (Thus, $\alpha^{2}=2 d_{+}-2 d_{-}+\chi$, where $\chi$ is the Euler characteristic of the normal bundle of $\Sigma \rightarrow S$.) Then

$$
d_{+} \geq\left(\alpha^{2}+1\right) / 4
$$

with equality only if $\alpha= \pm\left[E_{i}\right]$ for some $i$.

Notice that the conclusions in Propositions 4 and 5 refer only to the homology class of $\Sigma$, not to the isotopy class of the embedding or the regular homotopy class of the immersion.

Finally, as a corollary of Proposition 4, we obtain conjecturally the correct answer to the problem posed by Severi that was described at the end of the last section.

Proposition 6. Suppose Conjectures 2 and 3 hold for all simply connected algebraic surfaces $S$ with $p_{g}(S)=0$ and $\kappa(S) \geq 0$. If $X$ is an algebraic surface diffeomorphic to a rational surface, then $X$ is a rational surface.

Proof. If $X$ is diffeomorphic to a rational surface, then $b_{2}^{+}(X)=1$ and hence $p_{g}(X)=0$. Of course, $X$ is simply connected. If $X$ is not rational, then $\kappa(X) \geq 0$. Hence, by the hypotheses of Proposition 6 , Conjectures 2 and 3 hold for $X$, and its blow-ups. Thus, Proposition 4 holds for $X$. Hence, $X$ has no embedded 2-spheres of positive self-intersection. This implies that $X$ is not diffeomorphic to a rational surface, contrary to assumption.

Note. By the results of [17] for blown up Dolgachev surfaces it suffices to check Conjectures 2 and 3 for a finite set of minimal surfaces of general type and their blow-ups.

In order to state Conjectures 2 and 3 and their generalizations, we need the existence of a unique minimal model and hence the hypothesis $\kappa(S) \geq 0$. It is of interest to consider the case $\kappa(S)=-\infty$ as well and to try to find analogous results. If $S$ is moreover simply connected, then it is rational, and 
we are essentially reduced to considering $X_{k}$ equal to $\mathbf{C} P^{2} \# k \overline{\mathbf{C P}}^{2}$. In this case we have:

THEOREM (WALL [43]). If $k \leq 9$, every integral isometry of $H^{2}\left(X_{k} ; \mathbf{Z}\right)$ is induced by an orientation-preserving self-diffeomorphism of $X_{k}$.

Using the methods of Donaldson, one can investigate the isometries of $H^{2}\left(X_{k} ; \mathrm{Z}\right)$ for all $k$ and characterize those induced by self-diffeomorphisms of $X_{k}$. It turns out that they all "come from algebraic geometry," in some sense. However, for $k \geq 10$, they form a subgroup of infinite index in the full integral isometry group! (See [17] for more details.)

One can also consider non-simply connected surfaces with $\kappa(S)=-\infty$, i.e. ruled surfaces. Let $S$ be a ruled surface over $C$, with $g(C) \geq 1$. Suppose that $S$ is obtained from $S_{\min }$ by blowing up distinct points $x_{1}, \ldots, x_{k}$ (for the purposes of topology, we may always make this assumption). Let $e_{1}, \ldots, e_{k}$ be the cohomology classes dual to the exceptional curves, and $[F]$ the cohomology class dual to $\pi^{-1}(x)$, where $x$ is a general point of $C$. Thus $[F]^{2}=0$.

THEOREM 7. (a) If $\Sigma \subseteq S$ is an embedded 2-sphere and [ $\Sigma]$ is the cohomology class dual to $\Sigma$, then $[\Sigma]^{2} \leq 0$. Moreover, $[\Sigma]^{2}=0$ if and only if $[\Sigma]=n[F], n \in \mathbf{Z}$, and $[\Sigma]^{2}=-1$ if and only if $[\Sigma]= \pm e_{i}+n[F]$ for some $i$, $1 \leq i \leq k$, and some $n \in \mathbf{Z}$.

(b) Let $\varphi: S \rightarrow S$ be an orientation-preserving diffeomorphism. Then $\varphi^{*}([F])= \pm[F]$. Moreover, let

$$
A=\left\{\psi \text { an integral isometry of } H^{2}(S ; \mathbf{Z}): \psi([F])= \pm[F]\right\} .
$$

Then the elements of $A$ realized by self-diffeomorphisms form a subgroup of finite index in $A$.

We leave the proof as an exercise to the reader.

Arguments similar to those used to prove the above theorem give special cases of Conjectures 2 and 3 for certain surfaces with $\kappa \geq 0$ and "big" fundamental group, e.g. elliptic surfaces over curves of genus at least one or quotients of the unit ball in $\mathbf{C}^{2}$. The general principle at work, as in the discussion of Conjecture 1 , seems to be that the presence of a large fundamental group simplifies the topology of the surface.

4. Algebraic surfaces versus 4-manifolds. This section consists entirely of wild speculation restrained only by the few examples we know. The wildest speculation of all apparently goes back to René Thom.

SPECULATION. Is every simply connected 4-manifold $M$ diffeomorphic to $N_{1} \# N_{2} \# \cdots \# N_{k}$, where each $N_{i}$ is an algebraic surface (possibly with the orientation reversed)?

Here, $S^{4}$ is the empty connected sum, in good Bourbaki tradition. Note that this is a modest speculation: it includes the smooth Poincare conjecture in dimension 4 , as well as the claim that every positive definite simply connected 4-manifold is diffeomorphic to $n \mathrm{C} P^{2}$. Another consequence would be the following: If $M$ is simply connected and $b_{2}^{+}(M)$ and $b_{2}^{-}(M)$ are both even, then $M$ is decomposable. Yet another consequence would be the following: For $k \leq 8$ the topological manifold $C P^{2} \# k \overline{\mathbf{C P}}^{2}$ has only finitely many 
distinct smooth structures. We leave it to the reader to judge the plausibility of this speculation. A weaker version of this speculation is:

CONJECTURE 8. Every simply connected 4-manifold is homotopy equivalent to one of the form $N_{1} \# N_{2} \# \cdots \# N_{k}$ as above.

There is some evidence for this. If the intersection form of $M$ is odd, then, by the algebraic classification of forms, either it is isomorphic to a diagonal form $\bigoplus_{n}\langle 1\rangle \oplus \bigoplus_{m}\langle-1\rangle$ or it is definite. By Donaldson's theorem [11], if the intersection form of $M$ is definite it is also diagonalizable. Since a 4-manifold with diagonal intersection form is homotopy equivalent to $n \mathbf{C P} \# m \overline{\mathbf{C P}}^{2}$, Conjecture 8 is true for odd intersection forms.

If the intersection form of $M$ is even, then this conjecture is equivalent to the "eleven-eighths" conjecture (due to Kas and Kirby):

THE 11/8 CONJECTURE. If $M$ is a simply connected 4-manifold with even intersection form, then

$$
8 b_{2}(M) \geq 11 \cdot|I(M)| .
$$

The fact that this conjecture holds for connected sums of algebraic surfaces is immediate from the fact that it holds for algebraic surfaces (the latter a fact apparently first noticed by Moishezon). Note that an algebraic surface with an even intersection form must be minimal. To prove the result for algebraic surfaces with $I(M) \geq 0$ one appeals to the Bogomolov-Miyaoka-Yau inequality [5] that for an algebraic surface $c_{1}^{2} \leq 3 c_{2}$. If $I(M)<0$, then one uses the fact that for minimal simply connected algebraic surfaces $c_{1}^{2} \geq 0$ (which follows from the Kodaira classification) and the fact that any even form of rank $<8$ has zero signature. Conversely, any simply connected 4-manifold $M$ with even intersection form satisfying $8 b_{2}(M) \geq 11 \cdot|I(M)|$ is homotopy equivalent to a connected sum of $|I(M)| / 16$ copies of the $K 3$ surface and $\left(8 b_{2}-11 \cdot|I(M)|\right) / 16$ copies of $S^{2} \times S^{2}$. (Note that $|I(M)| / 16$ is an integer because of Rokhlin's theorem and $8 b_{2}-11 \cdot|I(M)|$ is divisible by 16 by the algebraic classification of forms.)

Donaldson has recently shown [13] that if $M$ is simply connected with even intersection form and if $b_{2}(M)-|I(M)| \leq 4$ (i.e., if $\max \left(b_{2}^{+}(M), b_{2}^{-}(M) \leq 2\right.$ ) then $I(M)=0$ and $M$ is homotopy equivalent to a connected sum of $S^{2} \times S^{2}$ 's. This is a very special case of the $11 / 8$ conjecture.

Speculation of the above type must be restricted to simply connected 4manifolds, even if we replace algebraic surfaces by compact complex surfaces. For instance, by combining results of Bogomolov [7] and Inoue [23] (cf. also Kodaira [26]) one can show that if $\Sigma^{3}$ is a rational homology 3-sphere, then $S^{1} \times \Sigma^{3}$ is homotopy equivalent to a connected sum of compact complex surfaces only if $\Sigma^{3}=S^{3}$. (Complex structures do exist on $S^{1} \times S^{3}$, and these are all Hopf surfaces [26].) In fact, using the classification of surfaces one can construct many other examples.

Without being so ambitious in our speculation, we could simply restrict attention to those 4-manifolds which we get by taking connected sums of algebraic surfaces (possibly with the orientation reversed), and ask about 
the relations among these, i.e. when is $N_{1} \# N_{2} \# \cdots \# N_{k}$ diffeomorphic to $N_{1}^{\prime} \# \cdots \# N_{l}^{\prime}$, where the $N_{i}, N_{j}^{\prime}$ are algebraic surfaces or their negatives? A result due to Mandelbaum and Moishezon [29, 32] shows that cancellation fails to hold in a spectacular way. They show that, if $S(p, q)$ is the Dolgachev surface with multiple fibers of multiples $p$ and $q$, then for all $(p, q)$ and $\left(p^{\prime}, q^{\prime}\right) \in \mathbf{N} \times \mathbf{N}$ with g.c.d. $(p, q)=$ g.c.d. $\left(p^{\prime}, q^{\prime}\right)=1, S(p, q) \# \mathbf{C} P^{2}$ is diffeomorphic to $S\left(p^{\prime}, q^{\prime}\right) \# \mathbf{C} P^{2}$. In the same spirit, they showed in [30] that, if $V_{d}$ is a smooth hypersurface of degree $d$ in $\mathbf{C} P^{3}$, then $V_{d} \# \mathbf{C} P^{2}$ is diffeomorphic to a connected sum $a \mathbf{C} P^{2} \# b \overline{\mathbf{C P}}^{2}$, where $a$ and $b$ are functions of $d$. Earlier and more generally, Wall showed that if $M$ and $M^{\prime}$ are simply connected 4-manifolds with the same self-intersection form, then, after taking connected sum with sufficiently many $\mathbf{C} P^{2}$ 's and $\overline{\mathbf{C P}}^{2}$ 's, $M$ and $M^{\prime}$ become diffeomorphic. ([In [32], Moishezon gave an explicit upper bound on the number of $\mathbf{C} P^{2}$ 's and $\overline{\mathbf{C P}}^{2}$ 's needed in case $M$ and $M^{\prime}$ are algebraic surfaces.) So the relations among connected sums of algebraic surfaces seem to be very complicated. The general principle, however, seems to be that as soon as we leave the algebraic category via connected sums, the structure of the 4-manifold simplifies dramatically. Along these lines, Mandelbaum [29] conjectured that, if $M$ is a simply connected algebraic surface, then $M \# \mathbf{C} P^{2}$ is diffeomorphic to $r \mathrm{C} P^{2} \# s \overline{\mathbf{C P}}^{2}$. On the other hand, considerable efforts to decompose algebraic surfaces themselves into standard pieces failed. After this Mandelbaum and Moishezon [ $\$ 6$ of 30] conjectured that nonrational algebraic surfaces were never completely decomposable, i.e., were never diffeomorphic to a connected sum of $\mathbf{C} P^{2}$ 's and $\overline{\mathbf{C P}}^{2}$ 's. It was a triumph when Donaldson showed the following statement which implies this and much more in the case when $b_{2}^{+} \geq 2$ :

THEOREM (DONALDSON). Let $S$ be a simply connected algebraic surface, and suppose there is an orientation-preserving diffeomorphism from $S$ to $M_{1} \# M_{2}$, where $M_{1}, M_{2}$ are oriented 4-manifolds. Then one of $M_{1}, M_{2}$ has a negative definite self-intersection form.

Here is a possible complement to Donaldson's result, one which would say that the only possible decompositions of algebraic surfaces are the obvious ones coming from algebraic geometry.

SPECULATION. Suppose $S$ is an algebraic surface with $\kappa(S) \geq 0$ and suppose $\varphi: S \rightarrow M_{1} \# M_{2}$ is an orientation-preserving diffeomorphism with $M_{2}$ having a negative definite intersection form. Does there exist an algebraic surface $T$ and a blow-down $\rho: S \rightarrow T$ which realizes this decomposition? By this we mean the following: if $\rho: S \rightarrow T$ is a blow-down then there is a natural diffeomorphism $\iota: T \# k \overline{\mathbf{C P}}^{2} \stackrel{\cong}{\rightrightarrows} S$ well-defined up to isotopy. To say the blow-down realizes $\varphi: S \stackrel{\cong}{\rightarrow} M_{1} \# M_{2}$ means that there are diffeomorphisms $\psi: M_{1} \rightarrow T$ and $\psi^{\prime}: M_{2} \rightarrow \# k \overline{\mathbf{C P}}^{2}$ such that

$$
S \stackrel{\varphi}{\longrightarrow} M_{1} \# M_{2} \stackrel{\psi \# \psi^{\prime}}{\longrightarrow} T \# k \overline{\mathbf{C P}}^{2} \stackrel{\iota}{\rightarrow} S
$$

is isotopic to the identity. 
NOTE. Such results do not hold for rational surfaces.

As before, one can formulate a weaker version of this speculation which seems more accessible.

CONJECTURE 9. Suppose $S$ is a simply connected algebraic surface with $\kappa(S) \geq 0$. Suppose there is an orientation-preserving diffeomorphism $\varphi: S \rightarrow$ $M_{1} \# M_{2}$ with $M_{2}$ negative definite. Then there is a blow-down $\rho: S \rightarrow T$ such that $\varphi^{*} H^{2}\left(M_{1}\right)=\rho^{*} H^{2}(T)$ in $H^{2}(S)$, or equivalently with $\varphi^{*} H^{2}\left(M_{2}\right)$ equal to the span in cohomology of the exceptional fibers of $\rho$.

As a final note, those questions which we have called conjectures in this section pertain to the homotopy classification of smooth 4-manifolds and algebraic surfaces. The techniques of gauge theory seem to be a very promising approach to these problems. On the other hand, those questions which we have called speculations and which involve the diffeomorphism classification of 4-manifolds seem to require completely new ideas.

\section{REFERENCES}

1. L. Ahlfors and L. Bers, Riemann's mapping theorem for variable metrics, Ann. of Math. (2) 72 (1960), 385-404.

2. M. Atiyah, Geometrical aspects of gauge theories, Proc. Int'l. Congr. Math. (Helsinki, 1978), Acad. Sci. Fen., Helsinki, 1980, pp. 881-885.

3. M. Atiyah, N. Hitchin and I. Singer, Self-duality in four-dimensional Riemannian geometry, Proc. Roy. Soc. London 362 A (1978), 425-461.

4. R. Barlow, $A$ simply connected surface of general type with $p_{g}=0$, Invent. Math. 79 (1985), 293-301.

5. W. Barth, C. Peters and A. Van de Ven, Compact complex surfaces, Springer-Verlag, Berlin, Heidelberg, New York, Tokyo, 1984.

6. L. Bers, Quasi-conformal mappings and Teichmüller's theorem, Analytic Functions, (Nevanlinna et. al., eds.), Princeton Univ. Press, Princeton, N.J., 1960.

7. F. Bogomolov, Surfaces of class VII $\mathrm{VI}_{0}$ and affine geometry, Math. USSR-Izv. 21 (1983), 31-73.

8. E. Bombieri, Canonical models of surfaces of general type, Publ. Math. Inst. Hautes Études Sci. 42 (1973), 171-219.

9. P. Deligne, P. Griffiths, J. Morgan and D. Sullivan, Real homotopy theory of Kähler manifolds, Invent. Math. 29 (1975), 245-274.

10. I. Dolgachev, Algebraic surfaces with $p_{g}=q=0$, Algebraic Surfaces, C.I.M.E. 1977, Cortona, Liguori, Napoli, 1981, pp. 97-215.

11. S. Donaldson, An application of gauge theory to four dimensional topology, J. Differential Geom. 18 (1983), 279-315.

12. __ Anti-self dual Yang-Mills connections over complex algebraic surfaces and stable vector bundles, Proc. London Math. Soc. 50 (1985), 1-26.

13. Connections, cohomology, and the intersection forms of 4-manifolds, J. Differential Geom. 24 (1986), 275-341.

14.

15. __ Polynomial invariants for smooth 4-manifolds (in preparation).

16. M. Freedman, The topology of four-dimensional manifolds, J. Differential Geom. 17 (1982), 357-454.

17. R. Friedman and J. Morgan, On the diffeomorphism types of certain algebraic surfaces. I, II, J. Differential Geom. (to appear).

18. S. Glashow, Quarks with color and flavor, Sci. Amer. 223 no. 4 (1975), 38-50.

19. P. Griffiths and J. Harris, Principles of algebraic geometry, New York, Wiley, 1978. 
20. J. Harer, The second homology group of the mapping class group of an orientable surface, Invent. Math. 72 (1983), 221-240.

21. J. Harris and D. Mumford, On the Kodaira dimension of the moduli space of curves, Invent. Math. 67 (1982), 23-86.

22. R. Hartshorne, Algebraic geometry, Springer, New York, 1978.

23. M. Inoue, On surfaces of class $\mathrm{VII}_{0}$, Invent. Math. 24 (1974), 269-310.

24. K. Kodaira, On compact analytic surfaces. II, Ann of Math (2) 77 (1963), 563-626.

25. __ On the structure of compact complex analytic surfaces. II, Amer. J. Math. 88 (1966), 682-721.

26. __ Complex structures on $S^{1} \times S^{3}$, Proc. Nat. Acad. Sci. 55 (1966), 240-243.

27. M. Lübke and C. Okonek, Differentiable structures of elliptic surfaces with cyclic fundamental group, preprint.

28. F. Maier, Tulane Univ. thesis, 1987.

29. R. Mandelbaum, Four-dimensional topology: an introduction, Bull. Amer. Math. Soc. (N.S.) 2 (1980), 1-159.

30. R. Mandelbaum and B. Moishezon, On the topological structure of non-singular algebraic surfaces in $\mathrm{CP} P^{3}$, Topology 15 (1976), 23-40.

31. J. Milnor, On simply connected 4-manifolds, Proc. Internat. Sympos. Algebraic Topology, Mexico (1958), 122-128.

32. B. Moishezon, Complex surfaces and connected sums of complex projective planes, Lecture Notes in Math., vol. 603, Springer-Verlag, Berlin and New York, 1972.

33. C. Okonek and A. Van de Ven, Stable bundles and differentiable structures on certain elliptic surfaces, Invent. Math. 86 (1986), 357-370.

34. R. Penrose, The complex geometry of the natural world, Proc. Int'l Congr. Math., Helsinki (1978), 189-194.

35. V. Rokhlin, New results in the theory of four-dimensional manifolds, Dokl. Akad. Nauk. S.S.S.R. 84 (1952), 221-224.

36. J.-P. Serre, Cours d'arithmétique, Presses Universitaires de France, Paris, 1970.

37. D. Sullivan, Infinitesimal computations in topology, Publ. Math. Inst. Hautes Études Sci. 47 (1977), 269-332.

38. C. Taubes, Self-dual Yang-Mills connections on non-self-dual \&-manifolds, J. Differential Geom. 17 (1982), 139-170.

39. O. Teichmüller, Extremale quasikonforme Abbildungen und quadratische differentiale, Preuss. Akad. 22 (1939).

40. W. Thurston, On the geometry and dynamics of homeomorphisms of surfaces, preprint.

41. M. Ue, On the diffeomorphism types of elliptic surfaces with multiple fibers, Invent. Math. 84 (1986), 633-643.

42. K. Uhlenbeck, Removable singularities in Yang-Mills fields, Comm. Math. Phys. 83 (1982), 11-29.

43. C. T. C. Wall, Diffeomorphisms of 4-manifolds, J. London Math. Soc. 39 (1964), 141-149.

44. S. Weinberg, Unified theory of elementary particle interaction, Sci. Amer. 231, no. 1, (1974), 50-59.

45. J. H. C. Whitehead, On simply connected, 4-dimensional polyhedra, Comment. Math. Helv. 22 (1949), 48-92.

46. C. N. Yang and R. Mills, Conservation of isotopic spin and isotopic gauge invariance, Phys. Rev. 96 (1954), 191-195.

Department of Mathematics, Columbia University, New York, New YORK 10027 
